\title{
Les Tiques (Acarina, Ixodoidea) du Sud-Ouest de la France
}

\author{
par Michel LAMONTELLERIE
}

Nous passerons en revue ici les espèces capturées dans les départements suivants : Vendée, Deux-Sèvres, Vienne, Charente-Maritime, Charente, Haute-Vienne, Gironde, Dordogne, Landes, Lot-et-Garonne, Gers, Basses-Pyrénées et Hautes-Pyrénées (1).

Nous avons connaissance, à l'heure actuelle, de l'existence, dans les départements considérés, de 19 espèces d'Ixodoödes (Ixodidae : 16 - Argasidae : 3 ). Parmi ces espèces, deux sont de capture accidentelle (Amblyomma variegatum et Hyalomna aegyptium) et ne peuvent être considérées comme faisant partie de la faune locale : leur présence est cependant nécessaire à noter, car elle permet d'envisager la possibilité d'importation d'espèces nouvelles pouvant, le cas échéant, s'adapter à la région et faire partie intégrante de sa faune, comme le cas s'est produit, pensons-nous, durant la dernière guerre pour Hyalomna scupense, Tique du bétail.

\section{I. - IXODIDAE}

1. Amblyomma variegatum (Fabricius, 1794):

Espèce africaine dont la présence en France est accidentelle.

Trois mâles connus de Dordogne: Vitrac, Chien, 10-4-50 (Lamontellerie, 1954). Comme le fait remarquer Hoogstraal (1956), il est probable que ces exemplaires ont été importés, à l'état de larves ou de nymphes, par des oiseaux migrateurs.

\section{Dermacentor marginatus Sulzer, 1776 :}

Nous avions signalé cette espèce (Lamontellerie, 1954) du Sud-Ouest, sous le nom de D. niveus (Neumann, 1897); il s'agit en fait d'une espèce différente.

\section{RÉPARTTIION :}

— Deux-Sèvres : Niort (Enigk, 1947).

(1) Nous remercions vivement MM. Colas-Belcour, Marc-André et Chabaud qui nous ont permis de consulter les collections de l'Institut Pasteur de Paris, du Museum National d'Histoire Naturelle de Paris et de la Faculté de Médecine de Paris (Collection Brumpt), ainsi que M. Morel, qui nous a fourni des renseignements concernant la collection Neumann (Toulouse). 
- Vienne : Châtellerault (Enigk, 1947). Cette référence est à ajouter à la carte de répartition des Dermacentor de France, établie par Roman et Sicard (1957).

- Charente-Maritime : Royan, La Rochelle (Enigk, 1947). St-Thomas-de-Cônac, St-Ciers-du-Taillon, Le Guâ.

— Gironde: Arcachon, Cadillac, Gujan, Libourne (Enigk, 1947).

St-Symphorien (Coll. Inst. Pasteur, Paris, homme, 25-3-51, Rivière rec.).

Bordeaux, Gujan, Léognan, Mérignac, Portets, Villenave-d'Ornon.

- Haute-Vienne: Limoges (Enigk, 1947).

- Landes: Dax, Mont-de-Marsan, Labenne (Enigk, 1947).

Samadet (Coll. BRUMPT, bœuf, 17-5-30, Baradat rec.).

Arengosse, Bey-Longue, Morcenx, Rion-des-Landes, Ychoux, Sindères.

- Dordogne : Savignac-de-Miremont ; cette référence est à ajouter à la carte de Roman et Sicard (1957).

- Basses-Pyrénées: Biarritz (Enigk, 1947).

St-Jean-de-Luz (Coll. Neumann, bœuf, deux mâles et trois femelles, 1894).

- Lot: Bien que ce département déborde notre cadre, citons la capture de D. marginatus sur Sus scrofa L. (9-3-52), cette référence étant à ajouter à la carte de Roman et Sicard (1957).

Hôtes : Bœuf (a). (1).

Cheval (a).

Erinaceus europaeus L. ( $n$ ).

Chien $(a)$.

Sus scrofa L. (a).

Mouton (a).

Homme ( 3 mâles et 3 femelles).

\section{Notes biologiques :}

Cette espèce est fréquente sur les bovins chez lesquels elle se localise le plus souvent entre les cornes et à l'extrémité de la queue. Chez les ovins, se trouve généralement à l'extrémité de la queue. Nous ne l'avons rencontrée qu'une fois sur le chien.

Chez l'homme, la localisation se fait le plus souvent à la nuque et la piqûre s'accompagne généralement d'une réaction lymphangitique.

Cette espèce est facile à élever sur hérisson et lapin. La larve se gorge volontiers sur souris, rat et cobaye.

Adultes de fin janvier à début mai ; nymphes en septembre-octobre.

3. Dermacentor reticulatus (Fabricius, 1794):

Cette espèce est nommée D. pictus (Hermann, 1804) par de nombreux auteurs. Nous adoptons ici la terminologie utilisée par Arthur dans sa révision du Genre (1960).

\section{RÉPARTITION :}

- Vienne: Poitiers et environs (Enigk, 1947-Rageau, communication orale). Lathus (Coll. Brumpt, hôte et date non précisés).

- Charente-Maritime : Royan (Enigk, 1947).

Le Guâ, Reignac de Blaye, Sablonceaux, St-Ciers-de-Taillon, St-Thomas-deConac.

(1) Nous désignons par $a, n, l$, les captures d'adultes, de nymphes et de larves. 
- Charente: Cognac (Enigk, 1947).

- Haute-Vienne : Limoges (Enigk, 1947 et coll. personnelle).

- Gironde: Cité de ce département par Coléno (1924), Carré et Farbos (1935), il est cependant possible que l'espèce de ces derniers auteurs soit à rapporter à $D$. marginatus.

Libourne et Sud de Bordeaux (Enigk, 1947).

Bordeaux, Léognan, Mérignac, Pont-de-la-Maye, St-Jean-d'Illac.

- Dordogne: Journiac, Le Bugue, Savignac-de-Miremont, Tourtoirac, Vitrac.

Ces références sont à ajouter à la carte de Roman et Sicard (1957).

- Landes: Cité du département par Carré et Farbos (1935), même remarque que cidessus.

Dax, Labenne, Parentis, Sabres (Enigk, 1947).

Samadet (Coll. Brumpt, 11 mâles et 3 femelles, 17-5-30, Baradat rec.).

Morcenx, Roquefort, Sindères.

- Lot-et-Garonne : Marmande (Enigk, 1947).

Agen, Sainte-Bazeille.

- Basses-Pyrénées: Roman et Sicard (1957) indiquent que, d'après Enigk (1947), cette espèce a été trouvée dans ce département ; nous n'avons pas retrouvé cette référence.

- Gers : Roman et Sicard (1957).

Vic-Fézensac (Morel, lettre personnelle : une femelle, chien, 14-5-60).

HôTes :

C'est avant tout, à l'état adulte, une tique du chien, que nous avons rencontrée aussi sur :

Bœuf (a).

Mouton (a).

Chèvre $(a)$.
Vulpes vulpes L. (a).

Sorex araneus ScHuB. (a).

Homme (3 mâles et 3 femelles).

D. reticulatus est rare sur les bovins de la région et relativement fréquent sur les . ovins sur lesquels on le rencontre en nombre à peu près égal à celui de l'espèce précédente.

\section{Notes biologiques :}

Les adultes apparaissent en octobre et disparaissent en juillet. Le maximum de fréquence a lieu en février-mars, époque à laquelle on les rencontre en abondance dans certains bois (Léognan, Savignac-de-Miremont, Roquefort...). A partir de fin avril, les exemplaires récoltés sont rares. Nous avons capturé des nymphes en juillet (Savignacde-Miremont).

Cette espèce est facile à élever sur hérisson et lapin. La larve se gorge aisément sur souris, rat et cobaye.

La larve mue une vingtaine de jours après avoir quitté son hôte.

Dans les six cas que nous avons observés chez l'homme, la piqûre n'a donné lieu à aucun phénomène clinique appréciable.

4. Ixodes ricinus (Linné, 1758) :

Cette espèce, largement répandue en zones tempérées, est certainement présente dans tout le Sud-Ouest. 


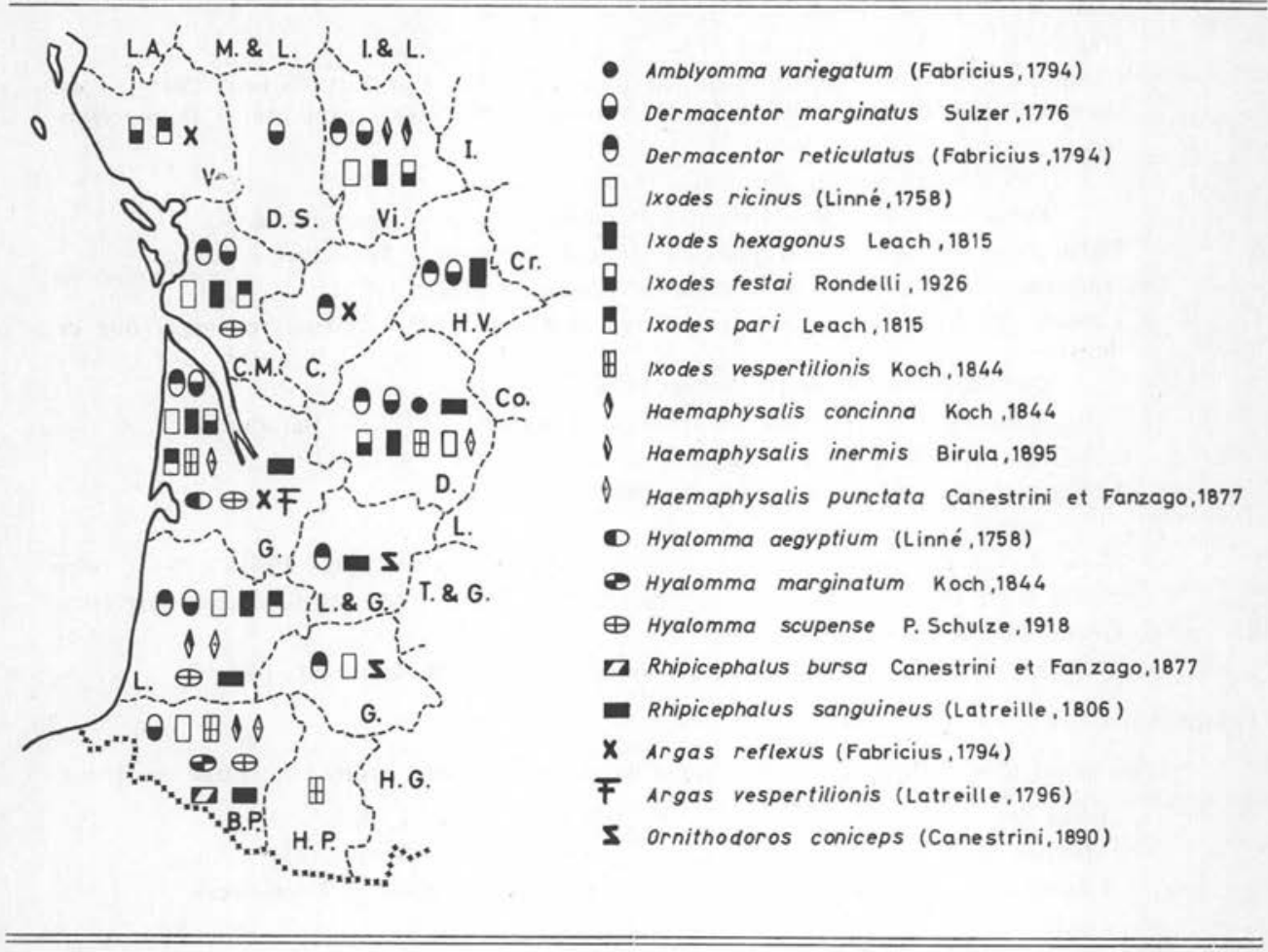

RÉPARTITION :

- Vienne: Poitiers (Coll. Institut Pasteur, Lepus timidus L., 23-10-59, une femelle, Rageau rec.).

- Charente-Maritime : Le Guâ, St-Ciers-du-Taillon, St-Thomas-de-Cônac.

- Gironde : Coléno (1924) cite cette espèce du département.

Taussat (Coll. Muséum, mousses, un mâle, août 47, Vachon, rec.).

Bazas, Bernos, Bordeaux, Cazaux, Gazinet, Gujan, Léognan, Portets, St-Jeand'Illac.

- Landes: Samadet (Coll. Brumpt, bœuf, une femelle, 18-7-30, Baradat rec.).

« Chalosse 》 (Coll. Brumpt, 9 mâles et 18 femelles, bœuf, juin 30, Baradat rec.).

Arengosse, Bey-Longue, Morcenx, St-Yaguen.

— Dordogne : Savignac-de-Miremont, Tourtoirac.

- Basses-Pyrénées: St-Jean-de-Luz (Coll. Neumann, une femelle, mouton, 1894).

St-Armou.

- Gers : Vic-Fézensac (Chien, 14-5-60, une femelle, Morel, lettre personnelle).

HôTES : Bœuf (a).

Chien (a).

Cheval (a).
Erinaceus europaeus L. $(1, n)$.

Lepus timidus L. (a).

Homme ( 1 femelle et 7 nymphes). 


\section{Notes biologiques :}

Nous avons capturé des adultes de février à octobre ; des nymphes de mars à octobre.

Cette espèce est facile à élever sur hérisson, à la température du laboratoire.

La larve se fixe aisément sur souris, rat et cobaye.

La ponte a lieu d'avril à juin. L'éclosion se fait de 17 à 22 jours après le début de la ponte. La larve peut vivre à jeun jusqu'à 350 jours et est capable de se gorger après plus de 300 jours de jeûne : il est fort probable que, dans la nature, sa longévité puisse aussi être très grande et que, de ce fait, le cycle complet puisse s'effectuer sur plusieurs années.

Quel que soit l'âge de la larve, la durée de son gorgement est à peu près constante : 5 à 9 jours. La larve gorgée quitte son hôte et la période de latence larve gorgée-nymphe est de 41 à 47 jours.

La nymphe, après s'être gorgée, quitte son hôte et il intervient alors un phénomène remarquable, concernant la période de latence nymphe gorgée-adulte :

a) Des nymphes, mises à gorger de mars à juillet (époque où elles sont fréquentes dans la nature) ont une période de latence de 34 à 40 jours et muent d'avril à juillet.

b) Par contre, des nymphes conservées à jeun, mises à gorger à partir d'août, se gorgent, quittent leur hôte, passent l'hiver sans muer et ne donnent des adultes que de mars à juillet de l'année suivante: Leur période de latence varie alors de 221 à 256 jours.

Il est probable que le même phénomène puisse avoir lieu dans la nature.

5. Ixodes hexagonus Leach, 1815 :

\section{RÉPARTITION :}

- Vienne: Champigny-Le-Sec (Rageau, communication orale).

Vivonne.

- Charente-Maritime : Saintes (Coll. Neumann, putois ( 5 femelles) et fouine ( 1 femelle), 1896, Lamoureux rec.).

La Villedieu (Coll. Brumpt, août-sept. 1918, nombreuses femelles).

Le Guâ, Sablonceaux, Saintes, St-Agnan.

- Haute-Vienne : Aixe-sur-Vienne.

- Gironde : Coléno (1924) cite cette espèce du département.

Pessac, Villenave-d'Ornon.

— Dordogne: Périgueux, Savignac-de-Miremont, Sigoulès, St-Alvère, Vitrac.

- Landes : Mont-de-Marsan (Coll. Institut Pasteur, chien).

Hôtes : Chien $(a, n, 1)$.

Chat $(a, n, 1)$.

Fouine (a).
Erinaceus europaeus L. $(a, n)$. Mustela putorius L. $(a, n)$.

\section{Notes biologiques :}

Adultes toute l'année, en particulier de mars à octobre. Nymphes d'avril à octobre. Larves de mai à décembre. 
Espèce facile à élever sur hérisson. La ponte a lieu en octobre et il semble que la période de chute de la femelle gorgée et fécondée n'amène pas de variation dans la période de ponte : deux femelles ayant quitté leur hôte respectivement les 25 mars et 25 juillet ont commencé de pondre le 7 octobre.

Les œufs éclosent de 68 à 78 jours après le début de la ponte.

6. Ixodes festai Rondelli, 1926 :

- Vendée: Croix de Vie (lapin de garenne, une femelle, Coll. Institut Pasteur. Cité par Morel, 1959).

- Vienne: Vivonne (chat, 23-3-1951, 2 mâles et 1 femelle).

- Gironde : Pont de la Maye, sur Sciurus vulgaris L.

- Dordogne : Saint-Alvère, 16-10-1960, 1 femelle, 15 nymphes et 8 larves, sur Erinaceus europaeus $\mathrm{L}$.

7. Ixodes pari Leach, 1815 :

Nous adoptons ici la terminologie utilisée par Arthur (1963) pour cette espèce dénommée par la plupart des auteurs Ixodes frontalis (Panzer, 1795). D'autre part, considérant la description d'Ixodes pari donnée par Arthur, Ixodes sigalasi, que nous avons décrit en 1954, tombe en synonymie avec Ixodes pari.

RÉPARTITION ET HÔTES :

- Vendée: Challans (Coll. Muséum, février 1945, Dresco rec., cité par Morel, 1959).

- Gironde: Villenave-d'Ornon, 22-3-52, deux nymphes, sur Erythacus rubecula L.

- Charente-Maritime : Saintes (une larve, sur Turdus merula L., citée par Neumann en 1899).

Montendre : une femelle, 5-1-53, sur Vanellus vanellus $\mathrm{L}$.

- Landes: Région de Dax, une femelle, 8-2-54, sur Turdus sp.

Signalons d'autre part que plusieurs chasseurs de Dordogne nous ont affirmé avoir vu des tiques fixées sur la nuque de grives, peut-être s'agit-il de cette espèce.

8. Ixodes vespertilionis Koch, 1844 :

- Gironde : Villenave-d'Ornon, 16-3-54, sur Myotis myotis (Bechst) (I).

13-8-51, sur Eptesicus serotinus (Schreber) (1).

- Basses-Pyrénées: Neumann (1916) signale cette espèce de diverses grottes: gr. de Ribenac (Arudy), gr. d'Iriberry (Iriberry), gr. de Compagnaga Lecia (CamouCihigne).

Arette (grotte d'Ambielle) : 3-3-1952. Coiffait rec. (Coll. Inst. Pasteur, Paris).

- Dordogne : Savignac-de-Miremont, oct. et nov. 1960, 1, n, a, sur Rhinolophus euryale Blasius.

- Hautes-Pyrénées : Signalé de plusieurs grottes par Neumann (1916) : gr. d'Ilhet (Ilhet), gr. de Gerde (Bagnères-de-Bigorre), gr. du Bas-Nistos (Haut et Bas-Nistos).

\section{Haemaphysalis concinna Koch, 1844 :}

\section{RÉPARTITION :}

- Vienne : Indiqué de ce département par Nuttall et Warburton (1915). 
- Landes: «Chalosse » (Coll. Brumpt, bœuf, 10-4-31, nombreux adultes, Baradat rec.). Arengosse.

- Basses-Pyrénées : St-Jean-de-Luz (Coll. Neumann, un mâle, mouton, 1894).

Ahusguy (Coll. Muséum, oct. 1896, une femelle, déterminée par Neumann, M. Brölemann rec.).

Hôtes : Bœuf $(a, n)$.

Mouton (a).

10. Haemaphysalis inermis Birula, 1895 :

Espèce citée de la Vienne par Nuttall et Warburton (1915).

11. Haemaphysalis punctata Canestrini et Fanzago, 1877 :

RÉPARTITION :

— Gironde : Coléno (1924), Carré et Farbos (1935) signalent cette espèce du département. Bordeaux.

- Dordogne: Savignac-de-Miremont.

- Landes : Carré et Farbos (1935) signalent cette espèce du département.

Arengosse, Beylongue, Morcenx, Sindères.

- Basses-Pyrénées : St-Jean-de-Luz (Coll. Neumann, une femelle, bœuf, 1894, 5 mâles et 5 femelles, mouton, 1894).

HôTES: Bœuf (a).

Fauvette (Sylvia sp.) (1).

Mouton $(a, n)$.

Lepus europaeus Pallas (n).

\section{Notes biologiques :}

Adultes de février à juin; nymphes récoltées en avril et décembre; larves en juillet.

S'élève facilement sur hérisson. Il s'écoule 38 à 45 jours entre le début de la ponte et l'éclosion des œufs.

\section{Hyalomma aegyptium (Linné, 1758):}

Espèce de capture accidentelle, que l'on rencontre sur les tortues (Testudo graeca L.) importées d'Afrique du Nord. On peut la capturer chaque année chez les marchands d'animaux de Bordeaux (Gironde).

Marzinowsky (1927) cite la tortue Emys orbicularis L. comme hôte de cette espèce ; dans le Sud-Ouest, où cette tortue existe, nous n'avons pas trouvé $H$. aegyptium dans la nature.

\section{Hyalomma marginatum Koch, 1844 :}

Enigk (1947) signale cette espèce de Urt (Basses-Pyrénées), sur cheval; à priori cette référence ne semble pas impossible, puisque cette tique existe en Espagne (J. de Prada, Gil Collado et Mingo Alsina, 1951). Pour Morel (1959), il pourrait s'agir plutôt de $H$. lusitanicum Koch, 1844. 


\section{Hyalomma scupense P. Schulze, 1918 :}

Nous avions signalé cette espèce (Lamontellerie, 1954) du Sud-Ouest, sous le nom de $H$. savignyi (Gervais, 1844) $(=H$. marginatum $)$, en fait, il s'agit là d'une espèce différente.

De nombreux auteurs font tomber $H$. scupense P. Schulze, 1918, en synonymie avec $H$. detritum $P$. Schulze, 1919 ; nous n'adoptons pas cette manière de voir :

En effet, bien que ces deux « formes » diffèrent très peu morphologiquement (tout au moins en ce qui concerne le stade adulte), elles sont biologiquement différentes : $H$. scupense est une tique d'hiver, à un hôte; $H$. detritum est une tique d'été, à deux hôtes. Nous estimons que ces deux «formes » constituent deux espèces jumelles («sibling species» des auteurs de langue anglaise) et doivent conserver chacune son individualité, seule l'étude biologique permettant de rattacher une population donnée à une espèce ou à l'autre.

Morel (lettre personnelle) envisage le problème un peu différemment et considère que le complexe detritum-scupense ne comprend qu'une seule espèce; dans ce complexe existent plusieurs «variétés biologiques » :

- detritum, à deux hôtes, nymphe restant fixée jusqu'à la fin de l'hiver ;

- mauritanicum, à deux hôtes, nymphe détachée avant l'hiver et muant à terre ;

- scupense, à un seul hôte, nymphe muant sur l'hôte, avant l'hiver.

De toute façon, que l'on envisage la question d'une manière ou d'une autre, seule l'étude biologique permet une détermination précise (ce qui, en plus de l'intérêt « intellectuel » de la chose, peut présenter un intérêt épidémiologique, les diverses «formes » ne véhiculant pas obligatoirement les mêmes germes). Il est fort probable que le problème est comparable chez d'autres Ixodoïdes (en particulier des genres Hyalomma et Rhipicephalus).

\section{RÉPARTITION :}

- Charente-Maritime : Sablonceaux, St-Thomas-de-Cônac.

- Gironde: Gujan.

- Landes: Arengosse, Beylongue, Morcenx, Sindères.

- Basses-Pyrénées: Schulze (1950) a décrit de ce département H. Steineri enigkanium, qui est probablement à rattacher à $H$. scupense.

Nous pensons que cette espèce, jamais citée avant 1954, des départements du Sud-Ouest, malgré sa grande abondance, a été importée avec des chevaux par les troupes allemandes, entre 1940 et 1945 .

HôTES : Bœuf.

Cheval.

Deux exemplaires mâles, refixés, ont été récoltés, l'un sur un chien, l'autre sur un homme. 


\section{Notes biologiques :}

Adultes de février à avril, nymphes en février et mars : il s'agit donc bien là d'une espèce de saison froide, ce qui, biologiquement, indique que l'on a à faire à scupense.

Cette espèce peut s'élever sur hérisson, sur lequel elle se comporte comme une tique à un seul hôte, ce qui, biologiquement, est un second critère en faveur de scupense et non de detritum.

Nous n'avons pu l'élever ni sur lapin, ni sur souris, rat ou cobaye.

La ponte a lieu de fin mars à fin mai, le maximum des pontes ayant lieu durant la troisième semaine de mai.

L'éclosion a lieu de 45 à 68 jours après le début de la ponte, le maximum des éclosions ayant lieu fin juillet.

15. Rhipicephalus bursa Canestrini et Fanzago, 1877 :

Cette espèce, présente en Espagne, semble avoir été peu récoltée en France.

- Basses-Pyrénées: St-Jean-de-Luz (Coll. Neumann, 50 nymphes, mouton, 1894) ; Bayonne et environs (Enigk, 1947); St-Armou (bœuf, 26-6-54, coll. personnelle).

16. Rhipicephalus sanguineus (Latreille, 1806):

Il est probable que ce que nous désignons ici par $R h$. sanguineus groupe au moins deux espèces distinctes; nous reprendrons ultérieurement le problème, à la lumière de travaux en cours de la part de plusieurs auteurs.

Cette espèce, peu connue du Sud-Ouest, atteint par endroits une pullulation extraordinaire et nous en avons observé de véritables invasions domiciliaires à Tresses (Gironde), en mai 1951 et à Bordeaux (Gironde) en juin 1953.

\section{RÉPARTITION :}

- Gironde : Coléno (1924) cite cette espèce du département.

Sénevet (1937) indique que, d'après Brumpt, cette espèce serait absente du bordelais. Il semble cependant que son installation dans la région soit assez ancienne et nous possédons des exemplaires récoltés sur chien, à Bordeaux, en 1918 (Castebert rec.).

Bordeaux, Cap Ferrat, Draguignan, Eysines, Gujan, Jau, La Teste de Buch,

Le Bouscat, Pessac, Pont-de-la-Maye, Talence, Tresses, Villenave-d'Ornon.

- Dordogne : Savignac-de-Miremont, Tourtoirac, Vitrac.

- Landes: Cazaux (Coll. Institut Pasteur).

Arengosse, Bias.

- Lot-et-Garonne : Sainte-Bazeille.

- Basses-Pyrénées: Cambo (Enigk, 1947).

Hôtes : Chien $(a, n, 1)$.

Meles meles L. (a).

Chat $(a, n)$.

Erinaceus europaeus L. (a).

Lapin domestique $(a, n)$.

Vulpes vulpes L. $(a, n)$.

Malgré sa grande fréquence, nous ne l'avons jamais rencontré sur l'homme, dans la région (Sigalas et Lamontellerie, 1954). 
Notes biologiques :

Adultes de mars à septembre, avec maximum de fréquence en mai-juin.

A ces divers Ixodidae, capturés dans le Sud-Ouest, il y a lieu d'ajouter une espèce qui s'y trouve probablement, sa présence ayant été signalée dans des départements limitrophes :

17. Ixodes canisuga Johnston, 1849 : Récolté dans les départements proches suivants :

- Haute-Garonne: Décrit de Toulouse par Neumann (1901) sous le nom d'Ixodes hexagonus inchoatus.

Toulouse, 4 femelles: Coll. Neumann (cité par Morel, 1959).

- Indre : Forêt de Lancôme, 4 femelles, avril 1912, chien, coll. Brumpt (cité par Morel, 1959).

- Indre et Loire : St-Christophe (Coll. Brumpt, Vulpes vulpes L., 29-1-1885, ex coll. Blanchard).

\section{II. - ARGASIDAE}

1. Argas reflexus reflexus (Fabricius, 1794):

- Vendée : Ile-d'Yeu: Ker-Chalons (Coll. Muséum, 3-9-1903, Courteaux, rec.).

- Charente : Angoulême (Morel, communication orale).

- Gironde : Coléno (1924) cite cette espèce du département.

2. Argas vespertilionis (Latreille, 1796):

- Gironde: Bordeaux, 3 larves, septembre 1950, sur Pipistrellus kuhli L.

Villenave-d'Ornon, 2 larves, 16-11-60, sur Pipistrellus piptstrellus (Schreber).

(Coll. personnelle, cités par Colas-Belcour et Rageau, 1961 et 1962).

3. Ornithodoros coniceps (Canestrini, 1890):

- Lot-et-Garonne: La Croix Blanche, pigeonnier, 17-7-52 (Lamontellerie, 1960).

- Gers : Nombreux exemplaires $(a, n)$ dans les combles de l'église de Vic-Fézensac, le 14-5-60 (Morel, lettre personnelle). Cette référence est à ajouter à l'étude de la répartition de $O$. coniceps faite par Morel (1959).

\section{III. - AUTRES IXODOIDES DE LA FAUNE FRANÇAISE}

Bien que ces espèces n'aient pas été récoltées dans le Sud-Ouest, nous avons jugé utile de les citer ici, car il est possible que certaines d'entre elles puissent s'y rencontrer. Toutes appartiennent à la famille des Ixodidae :

18. Ixodes acuminatus Neumann, 1901 :

Espèce des Insectivores, citée par Morel, 1959.

19. Ixodes lividus $($ Koch, 1844) $=($ Ix. plumbeus Leach, 1815) :

Parasite des hirondelles de rivage, cité par Morel, 1959. 
20. Ixodes simplex simplex Neumann, 1906 :

Espèce des chauve-souris, citée par Arthur en 1956 et Beaucournu, en 1962.

21. Ixodes strigicola Schulze et Schlottke, 1929:

Parasite des Oiseaux, plus spécialement des nocturnes, cité par Morel, 1959.

22. Ixodes trianguliceps Birula, 1895:

Espèce des Rongeurs et Insectivores qu'on doit pouvoir rencontrer en France, elle a en effet été signalée en :

- Allemagne (Neumann, 1901, Ix. tenuirostris).

- Angleterre (Nuttall et Warburton, 1911).

- Belgique (Bernard, 1959).

- Danemark (Arthur, 1955).

- Italie (Starkoff, 1958).

- Russie (Pomerantzev, 1950).

- Suisse (Nuttall et Warburton, 1911).

23. Ixodes uriae White, 1852 :

Parasite de certains Oiseaux marins (macareux, guillemots, mouettes, puffins, etc.). Cité par Morel (1959).

24. Hyalomma lusitanicum Koch, 1844 :

Cité par Morel (1959).

\section{IV. - RESUME}

19 espèces d'Ixodoïdes ont été rencontrées dans le Sud-Ouest de la France, réparties en :

- Ixodidae : 6 genres, 16 espèces : Amblyomma (1, accidentel), Dermacentor (2), Ixodes (5), Haemaphysalis (3), Hyalomma (3, dont 1 accidentel), Rhipicephalus (2).

- Argasidae : 2 genres, 3 espèces: Argas (2), Ornithodoros (1).

Hyalomma scupense et $H$. detritum sont considérés ici comme deux espèces jumelles; Morel les considère comme des «variétés biologiques »

Il y a lieu d'ajouter à la carte de répartition des Dermacentor en France (Roman et Sicard, 1957), la présence de D. reticulatus en Dordogne et de D. marginatus dans la Vienne, la Dordogne et le Lot.

Il y a lieu d'ajouter à l'étude de Morel sur la répartition d'Ornithodoros coniceps (1959) le département du Gers, où cet auteur a capturé cette espèce. 


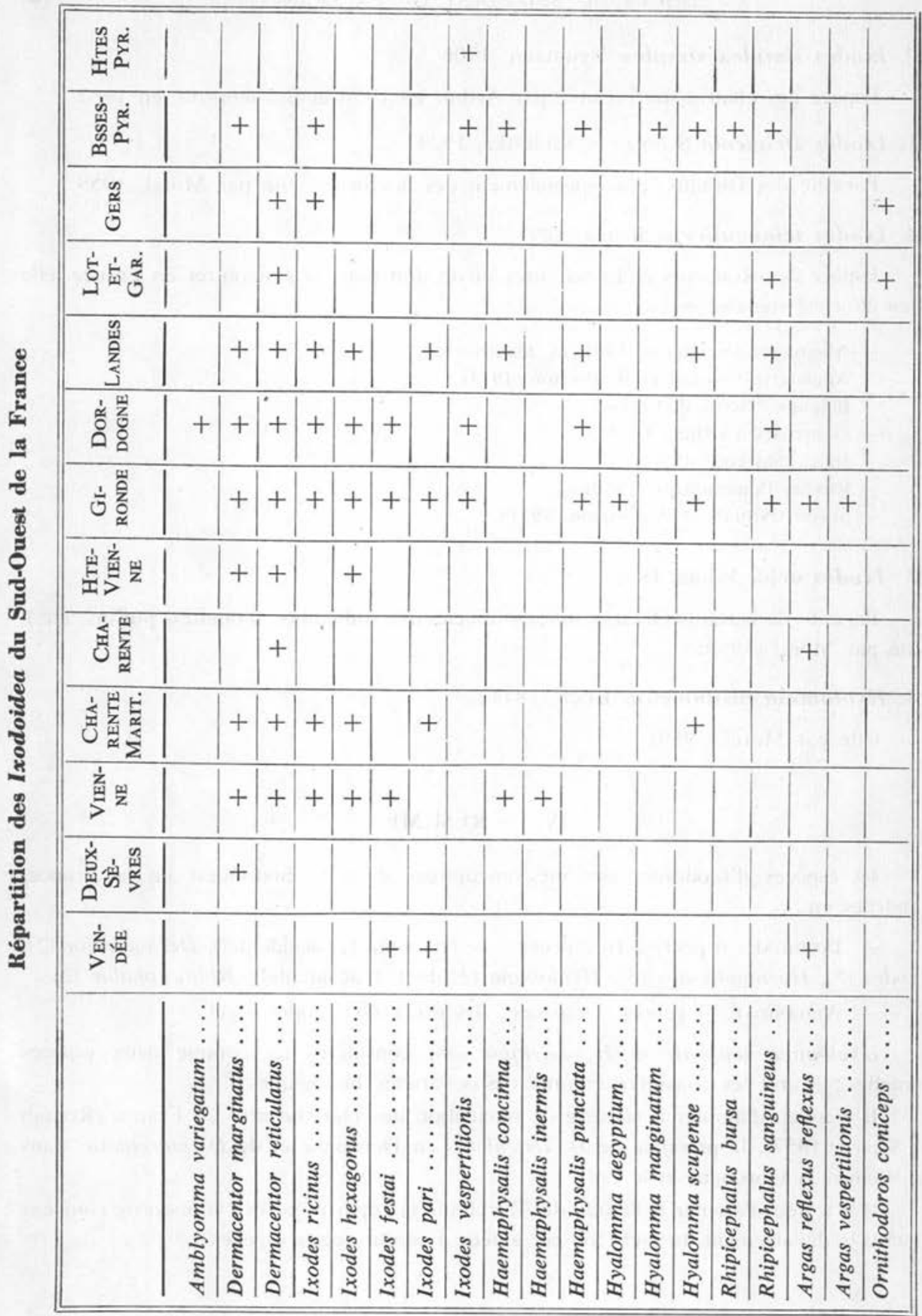




\section{Bibliographie}

Arthur (D. R.), 1952. - Ixodes frontalis Panzer, 1795. A review of the species. Parasitology, 42 (1-2), 151-154.

-, 1955. - Observations on collections of Ticks from Denmark. Ent. Med., 27, 76-81.

—, 1956. - The Ixodes ticks of Chiroptera (Ixodoidea, Ixodidae). J. Parasit., 42 (2), 180-196.

-, 1960. - Ticks. A monograph of the Ixodoidea. Part V: On the genera Dermacentor, Anocentor, Cosmiomina, Boophilus and Margaropus. Cambridge University Press, $251 \mathrm{pp}$.

-, 1963. - British Ticks, 213 pp. Butterworths ed. Londres.

Beaucournu (J.-C.), 1962. - Description du mâle d'Ixodes simplex simplex Neum., 1906, parasite de Chiroptères. Bull. Soc. Zool. Fr., 78 (1), 127-131.

BERnARD (J.), 1959. - Etudes sur les Métazoaires parasites du campagnol des champs: Microtus arvalis, Pall. (Rongeurs, Microtidae). I, Aperçu taxonomique. Bull. Inst. Agron. et des Stat. de Rech. de Gembloux, 27 (4), 371-407.

CARré (H.) et Farbos (J.), 1935. - Un foyer d'anaplasmose du mouton dans la région des Landes. C.R. Soc. Biol., 118 (1), 754-755.

Colas-Belcour (J.) et Rageau (J.), 1961. - Argasidae (Acariens, Ixodoidea) de France et d'Afrique du Nord. Arch. Inst. Pasteur, Maroc, 6 (2), 177-193 et Int. Kongr. Ent., Wien, 1960 Verhaudlungen. Bd. II. (Sekt. VII bis XIV), Wien, 1962.

Coléno, 1924. - Contribution à l'étude des Ixodidés et de leur rôle pathogène. Ixodidés de la Gironde. Th. Méd. Bordeaux.

ENIGK (K.), 1947. - Zur Kenntniss der Zecken von Süd-und Osteuropa. Mh. Vet. Med., 2 (5), 75-81.

Hoogstraal (H.), 1956. - African Ixodoidea. Vol. I : Ticks of the Sudan. Research Report NM 005, 050.29.07, U.S. Govt Print. Off., 1101 pp.

Lamontellerie (M.), 1954. - Ixodes sigalasi, n. sp., Ixodoide nouveau des Oiseaux. Ann. Parasit. hum. comp., 29 (5-6), 561-567.

—, 1954. - Les Ixodoïdes du Sud-Ouest de la France: Espèces rencontrées, agressivité, rôle pathogène. Th. Méd. Bordeaux, $145 \mathrm{pp}$.

—, 1960. - Présence d'Ornithodoros coniceps (Canestrini, 1890), dans le Lot-et-Garonne. Bull. Soc. Path. Exot., 53 (2), 159-160.

Marzinowsky (E.), 1927. - Du développement de l'Haemogregarina stepanovi. Ann. Parasit. hum. comp., 5 (2), 140-142.

Morel (P.-C.), 1959. - Sur quelques espèces peu communes du Genre Ixodes (Acariens, Ixodidae). Ann. Parasit. hum. comp., 34 (4), 546-548.

—, 1959. - Présence en France d'Ixodes festai Rondelli, 1926. Ann. Parasit. hum. comp., 34 (4), 549-551.

-, 1959. - Les Hyalomma (Acariens, Ixodidae) de France. Ann. Parasit. hum. comp., 34 (4), 552-555.

-, 1959. - Répartition de l'Ornithodoros coniceps (Canestrini, 1890). Ann. Parasit. hum. comp. 34 (5-6), 730-732. 
Neumann (L. G.), 1899. - Révision de la Famille des Ixodidés. Mém. Soc. Zool. Fr., 12 107-294.

—, 1901. - Révision de la Famille des Ixodidés. $4^{e}$ mémoire. Mém. Soc. Zool. Fr., 14, 249-372.

-, 1911. - Ixodidae, in Das Tierreich, Friedlander, Berlin.

-, 1916. - Biospeologica, 37. Ixodidei (Acariens), $1^{\text {re }}$ série. Arch. Zool. Exp. Gen., 55 (12), 515-527.

Nuttal (G.), Warburton (C.), Cooper (W.) et Robinson (L.), 1911. - Ticks, a monograph of the Ixodoidea. Part. II : The genus Ixodes. Cambridge University Press.

—, et Warburton (C.), 1915. - Ticks, a monograph of the Ixodoidea. Part III : The genus Haemaphysalis. Cambridge University Press.

Pomerantzev (B. I.), 1950. - Fauna S.S.S.R., 4 : Ixodidae. (Traduction anglaise de G. Anastos).

Prada (J. de), Gil Collado (J.) et Mingo Alsina (J. L.), 1951. - Tres Ixodidos Espanoles vectores de enfermedades rickettsiales. Jefatura de Sanidad de Burgos, mars, 35 pp.

Roman (E). et Sicard (M.), 1957. - Les Dermacentor de France (Acariens, Ixodidés). Bull. Soc. Hist. Nat., Toulouse, 92 (1-2), 161-170.

Schulze (P.), 1950. - Ueber Missbildungen der Schilzecken in Allbemeinen söne über Missbildungen von Hyalomma steineri enigkanium n. sp. in besondoren. Z. Parasitenk., 14 (6), 545-573.

Schultze (P.), 1950. - Ueber Missbildungen der Schilzecken in Allgemeinen söne über Miss-

Sigalas (R.) et Lamontellerie (M.), 1954. - Problèmes posés par l'épidémiologie de la fièvre boutonneuse. J. Méd. de Bordeaux, 131 (10), 963-968.

Starkoff (O.), 1958. - Ixodoidea d'Italia. Studio monografico, Rome, 339 pp.

(Laboratoire de Parasitologie - Faculté de Médecine et de Pharmacie de Bordeaux) 